\title{
DOMINANTLY INHERITED NEMALINE MYOPATHY
}

A locus on chromosome 15q21-23 for a dominantly inherited nemaline myopathy with core-like lesions is reported in two unrelated families evaluated at University Medical Center, Nijmegen, The Netherlands. TPM1 considered the strongest candidate gene had no identified disease-associated mutations. Patients have muscle weakness in neck flexors and proximal limb muscles without involvement of facial, respiratory, foot dorsiflexors or cardiac muscles. Muscle weakness is very slowly progressive and is manifested by the inability to perform fast movements such as running, and failure to break a fall when stumbling. Quadriceps biopsies showed type 1 fiber predominance with granular appearance suggestive of nemaline rods with Gomori trichrome stain. Zones devoid of ATPase and oxidative staining resembled central cores (pseudocores). Ultrastructural fiber examinations showed nemaline rods. (Gommans IMP, Davis M, Saar K et al. A locus on chromosome $15 \mathrm{q}$ for a dominantly inherited nemaline myopathy with core-like lesions. Brain July 2003;126:1545-1551). (Respond: BGM van Engelen MD PhD, Neuromuscular Centre, Nijmegen Institute of Neurology, University Medical Centre, PO Box 9101, 6500 HB Nijmegen, The Netherlands).

COMMENT. A novel nemaline core-like myopathy is described in two unrelated families with an uncharacterized genotype. Muscle weakness is manifested by slowness of movement and inability to break a fall.

\section{HEREDITARY MOTOR AND SENSORY NEUROPATHY WITH AGENESIS OF THE CORPUS CALLOSUM}

The epidemiological, clinical, and molecular genetic aspects of hereditary motor and sensory neuropathy and agenesis of the corpus callosum (HMSN/ACC) are reviewed by neurologists at McGill University, Montreal, Canada. Rarely reported worldwide, HMSN/ACC is prevalent in the Saguenay-Lac-St-Jean region of Quebec, Canada. In this area, the incidence at birth is 1 in 2,117 live births and the carrier rate is 1 in 23 inhabitants. A set of 22 founders originating from France was common to all of the cases. First described in 1966 (LeBlanc et al), the autosomal recessive inheritance was recognized in 1972 (Andermann et al). The clinical manifestations have an early onset with delay in developmental milestones, a severe sensory-motor polyneuropathy with areflexia, agenesis of the corpus callosum, amyotrophy, hypotonia, cognitive impairment, and psychoses. The average age of walking is 3.8 years, scoliosis appears at age 10.4 years, the ability to walk is lost by 13.8 years, and the average age of death is 24.8 years (33 years in a more recent unpublished study by the authors). The gene defect is a mutation in SLC12A6, which codes for the cotransporter protein KCC3. (Dupre N, Howard HC, Mathieu $\mathrm{J}$ et al. Hereditary motor and sensory neuropathy with agenesis of the corpus callosum. Ann Neurol July 2003;54:9-18). (Respond: Dr Guy A Rouleau, Centre for Research in Neurosciences and Department of Neurology and Neurosurgery, McGill University, 1650 Cedar Ave, Montreal, Quebec, H3G IA4 Canada). 